\title{
Metformin: Its emerging role in oncology
}

\author{
Dragan Micic ${ }^{1}$, Goran Cvijovic ${ }^{1}$, Vladimir Trajkovic ${ }^{2}$, \\ Leonidas H. Duntas ${ }^{3}$, Snezana Polovina ${ }^{4}$
}

${ }^{1}$ Institute of Endocrinology, Diabetes and Diseases of Metabolism, ${ }^{2}$ Institute of Microbiology and Immunology, Faculty of Medicine, University of Belgrade, Serbia, ${ }^{3}$ Endocrine Unit, Evgenidion Hospital, University of Athens, Greece, ${ }^{4}$ Endocrine Unit, General Hospital Subotica, Subotica, Serbia

\begin{abstract}
Metformin is considered, in conjunction with lifestyle modification, as a first-line treatment modality for type 2 diabetes mellitus (DM). Recently, several clinical studies have reported reduced incidence of neoplastic diseases in DM type 2 patients treated with metformin, as compared to diet or other antidiabetic agents. Moreover, in vitro studies have disclosed significant antiproliferative and proapoptotic effects of metformin on different types of cancer. Metformin acts by activating AMP-activated protein kinase (AMPK), a key player in the regulation of energy homeostasis. Moreover, by activating AMPK, metformin inhibits the mammalian target of rapamycin complex 1 (mTORC1) resulting in decreased cancer cell proliferation. Concomitantly, metformin induces activation of LKB1 (serine/threonine kinase 11), a tumor suppressor gene, which is required for the phosphorylation and activation of AMPK. These new encouraging experimental data supporting the anti-cancer effects of metformin urgently require further clinical studies in order to establish its use as a synergistic therapy targeting the AMPK/mTOR signaling pathway.
\end{abstract}

Key Words: Breast cancer, Cancer therapy, Colon cancer, Diabetes, Glioma, Metformin

\section{INTRODUCTION}

According to the Consensus Statement of the American Diabetes Association and the European Association for the Study of Diabetes, metformin is, together with lifestyle adjustments, a first-line treatment for type 2 diabetes mellitus (DM). ${ }^{1}$ Moreover, type $2 \mathrm{DM}$ and insulin resistance are associated with

Address for correspondence:

Professor Dragan Micic, Tel.: +381-64-6402843;

Fax: +381-11-3065081, e-mail: micicd@Eunet.rs

Received 05-05-10, Revised 10-09-10, Accepted 10-11-10 an increased risk for development of cancer, with breast, colorectal, prostate and pancreas cancers being reported most frequently. ${ }^{2-6}$ Recently, a large number of observational studies have been published reporting a reduced incidence of neoplastic disease in diabetic patients treated with metformin. ${ }^{7-9}$

The aim of this comunication is to review the data relating metformin with cancer and the possible mechanisms involved. The prospects for metformin as an alternative treatment modality in various forms of cancer as well as its potential role in preventive oncology are also outlined. 


\section{PERTINENT EPIDEMIOLOGIC DATA RELATING METFORMIN TO CANCER THERAPY}

Evans et $\mathrm{al}^{7}$ in 2005 reported that, of 11,876 patients with newly diagnosed type $2 \mathrm{DM}, 923$ were admitted to hospital with malignant cancer occurring during the observation period (1993-2001). Metformin therapy was associated with a reduced risk for cancer in this group of patients (odds ratio for any exposure to metformin was 0.79 ). At the same time, a greater protective effect was observed with increasing duration of exposure to metformin as well as with total number of prescriptions dispensed.

Shortly thereafter (2006), Bowker et $\mathrm{al}^{8}$ reported that in a cohort of 10,309 people diagnosed with type $2 \mathrm{DM}$ and followed for about 5 years, those who were exposed to sulfonylureas or exogenous insulin were significantly more likely to die of cancer-related causes than subjects exposed to metformin. The cancer mortality rate in the metformin group was about two thirds of that in the sulfonylurea group. Moreover, the risk of cancer-related mortality was even greater for insulin exposure (90\% relative increase) than for sulfonylurea exposure (30\% relative increase).

In a recent report (2009) by Libby et al, ${ }^{9}$ metformin treatment was associated with a reduced risk for cancer. In their study, approximately 4000 diabetic patients treated with metformin and 4000 patients treated with other therapy (comparators) were analyzed. Cancer was diagnosed in $7.3 \%$ of the metformin users compared with $11.6 \%$ of comparators. Median time to cancer diagnosis was 3.5 years for metformin users compared to 2.6 years for comparators. Also, metformin users were at much lower risk for overall mortality and cancer-related mortality than their comparators. Specifically, 3.0\% of metformin users died of cancer compared with $6.1 \%$ of comparators. It was earlier suggested that the sulfonylureas (and insulin) increase circulating insulin levels and hyperinsulinaemia may promote carcinogenesis. ${ }^{10}$ An additional issue, which, however, is not the focus of this review, are recent reports indicating that therapy with insulin analogues is associated with an increased risk for cancer. ${ }^{11}$

Approximately $20 \%$ of women of reproductive age have polycystic ovaries on ultrasound scan, while up to $10 \%$ have symptoms consistent with the diagnosis of polycystic ovary syndrome (PCOS) ${ }^{12}$ Because insulin resistance and consequent hyperinsulinemia are important etiological factors for the development of PCOS, metformin has been recommended as first choice treatment in the management of reproductive disorders caused by PCOS..$^{13}$ Obesity, hyperandrogenism, infertility, hyperinsulinemia and elevated levels of growth factors, which occur frequently in PCOS, are also factors known to be associated with development of breast cancer. However, studies examining the relationship between PCOS and breast cancer have not always identified a significantly increased risk. ${ }^{14-16}$ In the first of these studies, a relative risk of 1.5 was calculated for breast cancer in a group of women with chronic anovulation, this however not being statistically significant. ${ }^{14}$ In one large prospective study in a cohort of more than 30,000 women, benign breast disease was reported 1.8 times more frequently in women with PCOS compared to controls, though no increased likelihood for breast cancer development was found. ${ }^{15}$ More recently, in a series of 786 women with PCOS in the UK, calculated standardized mortality rates (SMR) have shown increased SMR for breast cancer in PCOS patients compared to controls, with breast cancer being in fact the leading cause of death in this cohort. ${ }^{16}$

While an association between PCOS and breast carcinoma is yet to be confirmed, the increased risk for endometrial carcinoma in women with PCOS is virtually certain. ${ }^{17}$ Moreover, although it was speculated that women with PCOS might be at increased risk for development of ovarian carcinoma, the results of studies conducted to date are conflicting, most of them having a false study design. ${ }^{14,16}$

Bearing in mind that metformin's role in the prevention of type $2 \mathrm{DM}$ as well in the treatment of polycystic ovary syndrome has recently been established, ${ }^{18,19}$ it would be of especial interest to determine whether metformin therapy applied in PCOS patients is associated with reduced risk of cancer in women as compared to other forms of therapy (PPR oral contraceptives, antiandrogens).

\section{METFORMIN IN CANCER THERAPY: POSSIBLE MECHANISMS INVOLVED}

\section{Metformin and AMP-activated protein kinase}

As mentioned previously, metformin is recom- 
mended as first-line therapy for type $2 \mathrm{DM}$ and consequently represents the most frequently used drug in the treatment of this disease. ${ }^{1}$ Although the principal mechanism of metformin action is reduction of hepatic glucose production, improvement in peripheral insulin action and $\beta$-cell function, reduction of lipolysis in adipocytes and intestinal glucose absorption have also been demonstrated..$^{20}$ The molecular basis underlying these clinical effects have been evaluated in in vivo and in vitro studies and it has been shown that metformin (in clinically relevant concentrations) brought about suppression of the mitochondrial respiratory chain, ${ }^{21}$ increased insulin receptor tyrosine kinase activity, ${ }^{22}$ stimulation of translocation of GLUT 4 transporters to the plasma membrane $^{23}$ and activation of AMPK. ${ }^{24}$ The latest reports indicate that metformin could be a potential agent for both prevention and treatment of neoplastic disease and the AMPK system was proposed as a key target point for metformin action. ${ }^{6,25-30}$

AMPK is an intracellular energy sensor that is activated by raising AMP and acts by switching on ATP-generating catabolic pathways while switching off ATP-requiring processes. ${ }^{31} \mathrm{It}$ is in an inactive form unless it is phosphorylated by upstream kinases at a threonine residue (Thr-172), in response to cellular stresses that deplete cellular energy levels as well as increase AMP/ATP ratio (glucose deprivation, hypoxia, hyperosmotic stress, tissue ischemia, muscle contraction/exercise). ${ }^{32}$ The ability of AMPK to directly sense cellular energy renders it capable of ensuring that cell division, which is a highly energy-consuming process, proceeds only if cells have enough metabolic resources to support this process. ${ }^{33}$ Activated AMPK restores cellular energy levels by stimulation of catabolic processes such as glucose uptake and/or glycolysis and fatty acid oxidation. ${ }^{33}$ The antineoplastic activity of metformin via the AMPK system is initiated by the activation of AMPK under conditions of normal metabolic stress, i.e. exercise or contraction of skeletal muscle. ${ }^{34}$ Exercise triggers AMPK-related glucose uptake by the skeletal muscles in an insulin-independent manner, phosphorylates and inhibits glycogen synthase and increases fatty acid oxidation..$^{35}$ Randomized clinical trials have revealed reduction in the incidence of recurrence of colon and breast cancer in patients who undertake long-term exercise. ${ }^{36}$ Since long-term exercise seems to increase AMPK levels, it may be hypothesized that a lessening of the recurrence of these cancers could, to some degree, be mediated by AMPK action in inhibiting cell growth..$^{33}$

Tumor suppressor gene-LKB-1 is one of the essential factors for activation of AMPK via exercise and administration of metformin. ${ }^{37-39} \mathrm{LKB}-1$ is an upstream kinase of the AMPK pathway and is responsible for phosporylation of Thr-172 and activation of AMPK. AMPK could not be activated by metformin analogues in mammalian cells that lacked LKB-1 expression. ${ }^{40,41}$ In mice lacking expression of LKB-1, markedly reduced AMPK activity in the liver was observed as well as a lack of reduction in blood glucose by metformin..$^{38}$ In Peutz-Jeghers syndrome (PJS), characterized by multiple gastrointestinal polyps and increased risk of epithelial malignancies, including breast cancer, the LKB-1 gene is mutated..$^{33}$ The mutation leads to activation of the Wnt signaling pathway, suggesting a Wnt-signaling role in the pathogenesis of gastrointestinal neoplasms in PJS. ${ }^{42}$ The Wnt/ $\beta$-catenin signaling cascade is an important signal transduction pathway in human cancers; overactivation of this pathway was demonstrated in several forms of tumors (gliomas, breast and colon cancer) ${ }^{43,44}$ Activation of the AMPK system by metformin inhibits growth of tumor cells through three different pathways in a tissue-dependent manner; inhibition of mammalian target of rapamycin (mTOR) and fatty acid synthesis (FAS), as well as stimulation of the p53/p21 axis ${ }^{30}$ (Figure 1).

mTOR is a serine-threonine protein kinase that belongs to the phosphoinositide 3-kinase (PI3K)related kinase (PIKK) family. It is integrated in two

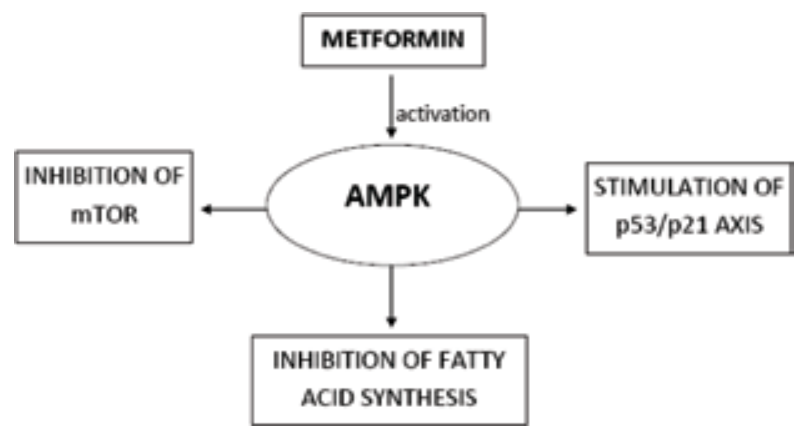

Figure 1. Metformin inhibition of tumor cell growth via AMPK. AMPK: AMP-activated protein kinase, mTOR: mammalian target of rapamycin. 
multiprotein complexes, TORC1 and TORC2, and is regulated by extracellular (insulin and insulin-like growth factors) and intracellular (nutrients, amino acids, glucose) signals, essential for cell growth. These growth factors and nutrients enhance mTORC1 function, this being followed by increased phosphorylation of ribosomal S6 kinase (S6K), a regulator of protein translation, while the key role of mTORC2 is the phosphorylation of the Akt/PKB. ${ }^{30}$ mTORC1 consists of mTOR, raptor (regulatory associated protein of mTOR) and mLST8, while mTORC2 consists of mTOR, rictor (rapamycin insensitive companion of mTOR), Sin-1 and mLST8. mTORC1 is regulated by nutrients and the PI3K/Akt signaling pathway via phosphorylation of the TSC2 protein. In addition to growth factor signals, the TSC1-TSC2 complex regulates mTOR activity. Phosphorylation of TSC2 by PI3K/Akt leads to inhibition of TSC2 and subsequent mTORC1 activation. In the absence of growth promoting stimuli, TSC 2 binds to TSC1 to form a tumor suppressor complex, which exerts growth-inhibitory activity via suppression of mTOR. ${ }^{45,46}$

mTOR is up-regulated in many cancer cells as a result of genetic alterations or aberrant activation of the components of the PI3K/Akt pathway, leading to dysregulation of cell proliferation, growth, differentiation and survival..$^{30,45}$ Aberrant activation of this pathway in breast cancer cells is through stimulation of epidermal growth factor receptor (EGFR), the estrogen receptor (ER), insulin and IGF1 receptors leading to cell proliferation and cancer progression. ${ }^{47}$ The clinical implications of mTOR activation are derived from the observation that invasive breast cancers overexpressive in mTOR have three times greater risk of recurrence and shorter disease-free survival. ${ }^{48}$ Experimental studies with metformin on epithelial cells demonstrated that metformin, through activation of the AMPK pathway, reduces cellular proliferation as a consequence of reduction of $\mathrm{mTOR}$ activation, S6K inactivation and general reduction of mRNA translation and protein synthesis. Activation of AMPK suppresses mTOR activation induced by growth factors and amino acids directly or indirectly via TSC $2 .{ }^{28,49}$

Rapamycin and its analogues exhibit antineoplastic activity and are now used clinically in the treatment of renal cell carcinoma. The antiproliferative effect of rapamycin is a consequence of mTOR inhibition, but it is limited in magnitude due to simultaneous inhibition of the mTOR-dependent feedback loop, leading to increasing signaling through IRS- 1 which results in increased AKT activation. Increased AKT activation stimulates cell survival pathways and inhibits apoptosis. Metformin also inhibits mTOR activation, but at the same time phosphorylates the Ser 789 inhibitory site of IRS-1 through AMPK activation and thereby reduces AKT activation. These findings suggest a more potent antineoplastic effect (antiproliferative + induction of apoptosis) of metformin by comparison with rapamycin. ${ }^{50}$

A second model suggesting the possible anti-cancer effect of metformin through the AMPK pathway is inhibition of fatty acid synthesis. ${ }^{30}$ Fatty acid synthesis is increased in many cancer cells, particularly breast cancer, as a result of high expression of fatty acid synthase (FAS), a key enzyme for fatty acid synthesis. ${ }^{51}$ High levels of FAS are associated with the malignant phenotype of breast and ovarian cancers, while inhibition of FAS suppresses cancer proliferation and induces cell death through apoptosis. ${ }^{30}$ Activation of AMPK via metformin leads to suppression of FAS gene expression and inactivation of acetyl-CoA carboxylase (ACC). This causes reduction in lipogenesis and synthesis of the ACC product malonyl-CoA resulting in increased fatty acid oxidation. ${ }^{52}$ This reduced expression of FAS and ACC results in suppression of prostate cancer cell proliferation. ${ }^{53}$

Finally, it has been suggested that AMPK activation promotes the survival of bioenergetically stressed stromal cells, in part through p53 activation. p53 is a tumor suppressor that is often mutated in cancer. In response to genotoxic stress, p53 induces a transcriptional response that can result in cell cycle arrest or apoptosis. At the same time, p53 demonstrated a prosurvival role in cells metabolically impaired by glucose deprivation. AMPK-dependent activation of p53 enables cells to arrest their proliferation until glucose is restored by redirection of metabolism to enhance $\beta$-oxidation of fatty acids and uptake of extracellular glucose.${ }^{54}$ In addition, p53, which plays an essential role in autophagy, the process that allows the cells to survive during deprivation of extracellular nutrients, can also be activated by metformin via the AMPK pathway. ${ }^{27,55}$ 


\section{MECHANISMS INVOLVED IN SPECIFIC TUMORS}

\section{Gliomas}

Gliomas are extremely aggressive neuroectodermal tumors and represent the most common primary malignancy in the human central nervous system. They are incurable in most cases and their resistance to apoptosis is suspected to contribute to chemotherapy and radiation resistance. Cell motility apparently contributes to the invasive phenotype of malignant gliomas, while interference with cell motility results in increased susceptibility of glioma to apoptosis. It was recently shown that metformin can inhibit in vitro migration of malignant glioma cells. Simultaneously, it was demonstrated that glioma cells express both AMPK $\alpha 1$ and AMPK $\alpha 2$ and that pharmacological activation of AMPK reduced glioma cell growth ${ }^{56,57} \mathrm{We}$ have demonstrated that metformin exerts a dual cell density-dependent anticancer action manifested either as a cell cycle arrest or caspase-dependent apoptotic death in low-density or high-density glioma cells. ${ }^{26}$ In low-density glioma cells, metformin inhibited the increase in glioma cell number in a dose-dependent manner and the highest concentration of the drug $(8 \mathrm{mM})$ completely blocked the proliferation of glioma cells. The proportion of cells in the $\mathrm{G}_{0} / \mathrm{G}_{1}$ cell cycle phase was significantly increased in metformin-treated glioma cultures, this suggesting that the antiglioma effect of metformin was mainly a consequence of cell cycle arrest. This antiproliferative effect was reversible: after withdrawal of the drug, glioma cells regained their proliferative capacity. Simultaneously, in confluent glioma cells after 48 hours of incubation with metformin, the initial number of the glioma cells was reduced to $<30 \%$ of control values. In this group of glioma cells, metformin induced activation of the AMPK pathway via downstream activation of the MAPK family member JNK. This activation leads to mitochondrial membrane depolarization and subsequent release of the small molecules such as cytochrome $\mathrm{c}$ that activate the caspase cascade and apoptosis. Collapse of the mitochondrial membrane generates ROS, providing a positive feedback mechanism and leading to further mitochondrial and cell injury. An interesting finding was that removal of glucose from the culture cell medium reduced the proapoptotic capacity of metformin, this further sug- gesting that glycolytic products rather than glucose deficiency due to excessive glycolysis contribute to metformin-induced glioma cell death. In both lowdensity and confluent glioma cells, primary astrocytes were completely resistant to the antiproliferative and apoptotic effect of metformin. ${ }^{26}$

At the same time, it was reported that the pharmacological inhibition of AMPK activity might be potentially useful in the treatment of certain types of cancers. Tumor xenografts lacking AMPK lost their ability to grow in a hypoxic environment, ${ }^{58}$ and $\mathrm{PC} 12$ pheochromocytoma cells transfected with dominantnegative AMPK underwent apoptosis upon glucose deprivation. ${ }^{59}$ Compound $\mathrm{C}$ (pyrrazolopyrinidine derivate) is a selective ATP-competitive inhibitor of AMPK that induced apoptosis in myeloma cell lines in the absence of any stress. ${ }^{60}$ We therefore evaluated the effect of AMPK inhibition on the human glioma cell line (U251) using compound C. ${ }^{61}$ Compound C decreased both the cell number and mitochondrial dehydrogenase activity in a dose-dependent manner. There was a considerable change in morphology of treated cells, suggesting cell death. In fact, the exposure to compound $\mathrm{C}$ induced cell cycle arrest in the G2/M phase and was associated with an increased number of apoptotic, hypodiploid cells with fragmented DNA (sub-G0/G1). Apoptosis of treated U251 cells was mediated by activation of caspases, including caspase- 3 and caspase- 8 . When the glioma cells were treated with compound $\mathrm{C}$ and metformin alone or in combination, compound $\mathrm{C}$ reduced AMPK activation by $30 \%$, while metformin markedly augmented AMPK activation and completely prevented compound C-induced inhibition of AMPK activation. Similar results were observed when we used another AMPK activator, AICAR.

Taken together, in vitro inhibition and stimulation of AMPK activity suggested an important role for this system in the evaluation of possible future treatment of gliomas. Further studies are required to ascertain which of these processes possesses the greatest potential and efficacy in the inhibition of proliferation and induction of apoptosis of glioma cells.

\section{Breast cancer}

Epidemiological studies over the past decade have shown an increased risk for development of breast 
cancer in patients with type $2 \mathrm{DM} .{ }^{2}$ Cohort and case control studies indicate that type $2 \mathrm{DM}$ enhances the relative risk (RR) of breast cancer by 10 to $20 \% .^{62}$ Insulin and IGF-1 receptors are relatively ubiquitous in breast cancers of all subtypes and patients with high insulin levels have a poor outcome, this suggesting that insulin and related ligand-induced signaling are highly associated with breast carcinogenesis. ${ }^{63} \mathrm{MCF}-7$ breast cancer cells were demonstrated to be responsive to insulin and insulin-like growth factors. ${ }^{64,65}$ Incubation of these cells with metformin induced activation of AMPK in a dose-dependent manner. Activation of AMPK was associated with inhibition of the mTOR pathway and decreased phosphorylation of S6K. Inhibition of AMPK using siRNA blocked the antiproliferative effect of metformin, implying that metformin exerts inhibition of cell growth directly and only through the AMPK pathway. ${ }^{28}$

An important issue within the context of the effect of metformin on breast cancer is triple negative (TN) breast cancer (also called basal), consisting of cells with no expression of the steroid receptors [ER and progesterone (PR)] as well as of the tyrosine kinase receptor Her-2, while overexpressing the EGFR. Epidemiological data have shown that TN cancers are more frequent in pre- and postmenopausal women with an elevated waist-hip ratio and body mass index, possibly signifying coexisting hyperinsulinemia and a positive therapeutic effect of metformin. ${ }^{66,67}$ Additionally, signaling pathway abnormalities commonly reported in this type of breast cancer involved p21mediated cell signaling and G1-S checkpoint controls that further suggest the possible therapeutic role of metformin in this group of breast cancer patients. ${ }^{68}$ In a comprehensive study by Liu B et al, TN breast cancer cells were found to be ultra-sensitive to metformin treatment, thus pointing to the potential of this drug in the future treatment of breast cancer. One in vitro component of this study revealed that metformin inhibited cellular proliferation in all tested $\mathrm{TN}$ breast cancer cells (4 types) in a dose-dependent manner. The striking issue is that this inhibition was obtained in concentrations that are within therapeutic range for patients taking metformin for type $2 \mathrm{DM}$. This inhibition of proliferation was associated with cycle arrest in G1 phase, increased percentage of cells in $\mathrm{S}$ phase and reduction of the fractions of cells in $\mathrm{G} 1$ and $\mathrm{G} 2 / \mathrm{M}$ phases together with reduction of cyclin D1 and cyclin E levels. Moreover, a sub-G1 peak was verified, indicating the induction of apoptosis. Further evaluation revealed that metformin induced apoptosis in $\mathrm{TN}$ breast cancer cells using both extrinsic (operating through caspase-8) and intrinsic (operating through caspase-9) pathways of caspase cascades. In another study, metformin-treated mice showed significantly slower growth of tumor and longer survival with tumors less than $2 \mathrm{~cm}$ in diameter. Immunohistochemical analysis confirmed a lower Ki67 index in metformin-treated compared to control mice. ${ }^{69}$

Alimova et al evaluated the effect of metformin on breast cancer cells using the most common subtypes of human breast cancer: luminal $\mathrm{A}\left(\mathrm{ER}^{+}\right.$, erbB2 $\left.{ }^{-}\right)$, luminal B $\left(\mathrm{ER}^{+}\right.$, erbB2 $\left.{ }^{-}\right)$and Her2/erbB2 (ER ${ }^{-}$, erbB2 $\left.{ }^{+}\right) \cdot{ }^{70}$ In all studied cell lines, metformin induced significant growth inhibition in a dose-dependent manner as well as inhibition of colony formation, both via an apoptosis-independent mechanism. These effects were associated with significant reduction in both cyclin D1 and E2F1, proteins that promote G1-S cell cycle translation. At the same time, metformin induced changes in receptor tyrosine kinase signaling, reduction in expression of erbB2 protein and activation of mitogen-activated protein kinase (MAPK). This effect was also dose-dependent and it was evident at metformin concentrations that are within the therapeutic range for patients with type 2 DM $(6-30 \mu \mathrm{M})$.

Most of the breast cancer cell lines are sensitive to metformin-induced growth inhibition but not all. ${ }^{71}$ There is no correlation between sensitivity to metformin and the known status of ER expression, p53 mutation or amplification of Her-2. ${ }^{72}$

Differences between breast cancer cell lines with regard to their response to metformin treatment were also reported by Phoenix et al. ${ }^{73}$ They found that the metformin effect was differentially related to either positive or negative estrogen receptors: the ER-negative cells are not as sensitive as are the ER-positive. In fact, ER-positive cells underwent complete growth inhibition, while ER-negative cells had only a partial inhibition.

These studies created a very good basis for the 
initiation of clinical studies with metfomin as a chemopreventive and therapeutic agent in women with breast cancer. Very recently, Jiralerspong et al reported a three-fold greater complete pathologic response in diabetic patients with breast tumors taking metformin and undergoing neoadjuvant chemotherapy compared with diabetic patients with breast tumor not taking metformin. ${ }^{74}$ Furthermore, Garcia and Tisman have provided an additional explanation for this enhanced pathologic complete response, namely, that chronic metformin administration is associated with vitamin B12 deficiency which is known to increase tissue toxicity in patients receiving adjuvant chemotherapy with methotrexate or after $\mathrm{N} 2 \mathrm{O}$ anesthesia. ${ }^{75}$

\section{Colon Cancer}

A possible contribution of the AMPK pathway in the treatment of colon cancer has also been described. ${ }^{27,76,77}$ It was suggested that selenium and EGCG (epigallocatechin-3-gallate, one of the major compounds of green tea), through generation of ROS, activates the AMPK pathway, which subsequently abrogates COX-2 expression as well as COX-2 and Prostaglandin $\mathrm{E}_{2}$ production in cancer cells. ${ }^{76,77}$ Activation of the AMPK pathway with selenium and EGCG lead in vitro and in vivo to the inhibition apoptosis cancer cells as well to the reduction of solid xenogaft tumors. Furthermore, combination treatment employing selenium/EGCG together with standard chemotherapy agents markedly reduced tumor cell viability as compared to treatment with 5-FU or Etoposide alone. This is particularly important considering that these effects were demonstrated in chemo-resistant HT-29 colon cancer cells. ${ }^{76,77}$ The possible inclusion of AMPK in anticancer treatment of colon cancer was demonstrated in p53-deficient colon cancer cells. ${ }^{27}$ Systematic treatment with metformin, through activation of AMPK, inhibits tumor growth in p53deficient colon cancer cells in vivo, while there is no effect in p53-positive cancer cells. Further analysis of tumor tissue treated with metformin revealed clusters of apoptotic cells in p53-deficient cells, especially in border regions that were under nutrient limitation, while electron microscopy showed no increase in autophagosome-positive cells. By contrast, p53 positive cells demonstrated a significant increase in autophagosomes in nutrient deprived cells. ${ }^{27}$ It was speculated that $\mathrm{p} 53$, besides its role as a tumor sup- pressor, plays a prosurvival role in cells metabolically impaired by glucose limitation. Activation of p53 by glucose deprivation is AMPK-dependent, leading to beta oxidation of fatty acids, capturing of extracellular glucose and induction of autophagy. Recently, autophagy was reported to be an essential survival factor of tumor cells in the center of the tumors. ${ }^{78}$ Loss of p53 impairs the ability of cancer cells to respond to metabolic changes induced by metformin and to survive under conditions of nutrient deprivation. Treatment with metformin induced apoptosis in vivo in this type of cancer cells that are usually resistant to existing forms of chemotherapy or radiotherapy. ${ }^{27}$

\section{METFORMIN AND PHARMACOGENOMICS}

The antiproliferative and proapoptotic effect of metformin in in vitro studies was observed in concentrations that are usually seen in diabetic patients treated with metformin (approximately 1.5 grams per day). However, there is inter-individual variation in drug response and the role of drug-metabolizing enzymes and drug transporters are starting to form the focus of these research projects. Genetic polymorphisms have been identified in most transport proteins and many of them are now recognized as significant contributors to inter-individual variation in drug effects. Metformin uptake in the liver occurs mostly by OCTs (organic cation transporters, transporters on sinusoidal membrane), while its efflux is facilitated by multidrug and toxin extrusion transporter 1 (MATE1). ${ }^{79}$ There were significantly higher glucose and insulin levels during OGTT in healthy volunteers with reduced-function OCT1 polymorphism compared to healthy volunteers with OCT1 reference alleles (after metformin treatment). Moreover, there was significantly reduced hepatic accumulation and therapeutic response to metformin in mice with OCT1 deletion. These findings suggest that OCT1 mediates the first step in the response pathway of metformin and that genetic variation in OCT1 may modulate response to metformin in humans. ${ }^{80} \mathrm{In}$ addition, identification of OCT3, the novel transporter of metformin, raises the possibility that genetic variation in OCT1 alone may not be sufficient to result in reduced response to metformin in some patients. OCT3 may contribute to hepatic metformin uptake when OCT1 is functionally impaired.$^{81}$ Finally, the 
second highest OCT1 expression is in the kidneys, being five-fold higher than in the small intestine. As metformin is a hydrophilic organic cation which is eliminated by the kidneys in more than $98 \%$ of the absorbed dose, the OCT1 polymorphism might play a significant clinical role in the therapeutic effect of metformin. Tzvetkov et al analyzed genetic polymorphisms in OCT1, OCT2 and OCT3 and their effects on metformin clearance. They found that the OCT1 polymorphism induces low or absent reasorption of metformin in distal tubules leading to increased metformin excretion, this possibly accounting for a $10 \%$ variation of metformin clearance. ${ }^{82}$

\section{PERSPECTIVES}

In vitro and in vivo studies strongly suggest that metformin could be a valuable adjuvant therapy in cancer treatment. It is noteworthy that the antineoplastic effect of metformin was observed in therapeutic concentrations achieved in patients with type 2 diabetes and that this effect was observed in cancer cells originating from different tissues: nervous system, breast, colon and prostate (Table 1). Most likely, during the next few years further basic research and more clinical studies will support, or reject, the hypothesis that metformin has an important role as an antineoplastic agent.

\section{Grants of fellowship supports}

This work was supported by the Serbian Ministry of Science (project number 145067).

Table 1. Reported effects of metformin on different types of cancer cell lines

\begin{tabular}{llll}
\hline Type of cancer & Proposed mechanisms of action & Author (ref. number) \\
\hline Breast & Arrest in G1 and S phases & Zakikhani M et al & $(28)$ \\
& Cyclin D1 and Cyclin E reduction & Liu B et al & $(69)$ \\
& MAPK and Akt inhibition & Alimova IN et al & $(70)$ \\
& mTOR and EGFR expression decrease & & \\
& S6 kinase inhibition & & \\
& Arrest in G1 phase & Isakovic A et al & $(26)$ \\
Gliomas & Activation of caspase cascade & Vucicevic L & $(61)$ \\
& Arrest in G1 phase & Ben Sahra I et al & $(29)$ \\
Prostate & Cyclin A and cyclin B reduction & Ben Sahra I et al & $(29)$ \\
& mTOR activity inhibition & Zakikhani M et al & $(50)$ \\
& Beta oxidation of fatty acids & Buzzai M et al & $(27)$ \\
Colon & Autophagy & Algire C et al & $(83)$ \\
& S6 kinase inhibition & & \\
\hline
\end{tabular}

\section{REFERENCES}

1. Nathan DM, Buse JB, Davidson MB, et al, 2006 Medical management of hyperglycemia in type 2 diabetes: a consensus algorithm for the initiation and adjustment of therapy: a consensus statement from the American Diabetes Association and the European Association for the Study of Diabetes. Diabetes Care 32: 193-203.

2. Saydah SH, Loria CM, Eberhardt MS, Brancati FL, 2003 Abnormal glucose tolerance and the risk of cancer death in the United States. Am J Epidem 157: 1092-1100.

3. Michels KB, Solomon CG, Hu FB, et al, 2003 Type 2 diabetes and subsequent incidence of breast cancer in the Nurses Health Study. Diabetes Care 26: 1752-1758.
4. Will JC, Galuska DA, Vinicor F, Calle EE, 1998 Colorectal cancer: another complication of diabetes mellitus? Am J Epidem 147: 816-825.

5. Everhart J, Wright D, 1995 Diabetes mellitus as risk factor for pancreatic cancer: a meta-analysis. JAMA 273: 1605-1609.

6. Gapstur SM, Gann PH, Colangelo LA, et al, 2001 Postload plasma glucose concentration and 27-year prostate cancer mortality (United States). Cancer Causes Control 12: 763-772.

7. Evans JM, Donnelly LA, Emslie-Smith AM, et al, 2005 Metformin and reduced risk of cancer in diabetic patients. BMJ 330: 1304-1305.

8. Bowker SL, Majumdar SR, Veugelers P, Johnson JA, 
2006 Increased cancer-related mortality for patients with type 2 diabetes who use sulfonylureas or insulin. Diabetes Care 29: 254-258.

9. Libby G, Donnelly LA, Donnan PT, Alessi DR, Morris AD, Evans JMM, 2009 New users of metformin are at low risk of incident cancer: a cohort study among people with type 2 diabetes. Diabetes Care 32: 1620-1625.

10. Moore MA, Park CB, Tsuda H, 1998 Implications of the hyperinsulinemia-diabetes-cancer link for preventive efforts. Eur J Cancer Prev 7: 89-107.

11. Hemkens LG, Grouven U, Bender R, 2009 Risk of malignancies in patients with diabetes treated with human insulin or insulin analogues: a cohort study. Diabetologia 52: 1732-1744.

12. Futterweit W, Mechanick JI, 1988 Polycystic ovarian disease: etiology, diagnosis, and treatment. Compr Ther 14: $12-20$.

13. Galluzzo A, Amato MC, Giordano C, 2008 Insulin resistance and polycystic ovary syndrome. Nutr Metab Cardiovas Dis 18: 511-518.

14. Coulam CB, Annegers JF, Kranz JS, 1983 Chronic anovulation syndrome and associated neoplasia. Obstet Gynecol 61: 403-407.

15. Anderson KE, Sellers TA, Chen PL, et al, 1997 Association of Stein-Leventhal syndrome with the incidence of postmenopausal breast carcinoma in a large prospective study of women in Iowa. Cancer 79: 494-499.

16. Pierpoint T, McKeigue PM, Isaacs AJ, et al, 1998 Mortality of women with polycystic ovary syndrome at long term follow up. J Clin Epidemiol 51: 581-586.

17. Balen A, 2001 Polycystic ovary syndrome and cancer. Hum Reprod Update 6: 522-525.

18. Diabetes Prevention Program Research Group, 2002 Reduction in the incidence of type 2 diabetes with lifestyle intervention or metformin. N Engl J Med 346: 393-403.

19. Ehrmann DA, 2005 Polycystic ovary syndrome. N Engl J Med 24: 1223-1236.

20. Campbell IW, Ritz P 2007 Understanding the glucoselowering actions of metformin. In: Bailey CJ, Campbell IW, Chan JCN, Davidson JA, Howlett HCS, Ritz P (Eds), Metformin The Gold Standard, John Wiley \& Sons, Ltd, Chichester, UK; pp, 77-88.

21. Detaille D, Guigas B, Leverve X, Wiernspreger N, Devos P, 2002 Obligatory role of membrane events in the regulatory effect of metformin on the respiratory chain function. Biochem Pharmacol 63: 1259-1272.

22. Giannarelli R, Aragona M, Coppelli A, Del Prato S, 2003 Reducing insulin resistance with metformin: the evidence today. Diabetes Metab 29: 628-635.

23. Matthaei S, Reibold JP, Hamman A, et al, 1993 In vivo metformin treatment ameliorates insulin resistance: evidence for potentiation of insulin-induced translocation and increased functional activity of glucose transporters in obese ( $\mathrm{fa} / \mathrm{fa}$ ) Zucker rats adipocytes. Endocrinology 133: 304-311.
24. Musi N, Hirshman MF, Nygren J, et al, 2002 Metformin increases AMP-activated protein kinase activity in skeletal muscle of subjects with type 2 diabetes. Diabetes 51: 2074-2081.

25. Bowker SL, Majumdar SR, Veugelers P, Johnson JA, 2006 Increased cancer-related mortality for patients with type 2 diabetes who use sulfonylureas or insulin. Diabetes Care 29: 1990-1991.

26. Isakovic A, Harhaji L, Stevanovic D, et al, 2007 Dual antiglioma action of metformin: cell cycle arrest and mitochondria-dependent apoptosis. Cell Mol Life Sci 64: 1290-1302.

27. Buzzai M, Jones RG, Amaravadi RK, et al, 2007 Systemic treatment with the antidiabetic drug metformin selectively impairs p53-deficient tumor cell growth. Cancer Res 67: 6745-6752.

28. Zakikhani M, Dowling R, Fantus G, et al, 2006 Metformin is an AMP kinase-dependent growth inhibitor for breast cancer cells. Cancer Res 66: 10269-10273.

29. Ben Sahra I, Laurent K, Loubat A, et al, 2008 The antidiabetic drug metformin exerts an antitumoral effect in vitro and in vivo through a decrease of cyclin D1 level. Oncogene 27: 3576-3586.

30. Hadad S, Fleming S, Thompson A, 2008 Targeting AMPK: a new therapeutic opportunity in breast cancer. Crit Rev Oncol Hematol 67: 1-7.

31. Carling D, 2004 The AMP-activated protein kinase cascade - a unifying system for energy control. Trends Biochem Sci 29: 18-24.

32. Hardie DG, Scott JW, Pan DA, Hudson ER, 2003 Management of cellular energy by the AMP-activated protein kinase system. FEBS Letters 546: 113-120.

33. Alessi DR, Sakamoto K, Bayascas JR, 2006 LKB1-dependent signaling pathways. Annu Rev Biochem 75: 137-163.

34. Hutber CA, Hardie DG, Winder WW, 1997 Electrical stimulation inactivates muscle acetyl-CoA carboxylase and increases AMP-activated protein kinase. Am J Physiol 272: E 262-266.

35. Winder WW, Hardie DG, 1999 AMP-activated protein kinase, a metabolic master switch: possible roles in type 2 diabetes. Am J Physiol 277: E1-10.

36. Willer A, 2003 Reduction of the individual cancer risk by physical exercise. Onkologie 26: 283-289.

37. Woods A, Johnstone SR, Dickerson K, et al, 2003 LKB1 is the upstream kinase in the AMP-activated protein kinase cascade. Curr Biol 13: 2004-2008.

38. Shaw RJ, Lamia KA, Vasquez D, et al, 2005 The kinase LKB-1 mediates glucose homeostasis in liver and therapeutic effects of metformin. Science 310: 1642-1646.

39. Thomson DM, Brown JD, Fillmore N, et al, 2007 LKB1 and the regulation of malonyl-Co A and fatty acid oxidation in muscle. Am J Physiol Endocrinol Metab 293: E1572-1579.

40. Hawley SA, Boudeau J, Reid JL, et al, 2003 Complexes between LKB-1 tumor suppressor, STRAD alpha/beta 
and MO25 alpha/beta are upstream kinases in the AMPactivated protein kinase cascade. J Biol 2: 28.

41. Hoyer-Hansen M, Jaattela M, 2007 AMP-activated protein kinase: a universal regulator of autophagy? Autophagy 3: 381-383.

42. Chaiyapan W, Sangkhathat S, Kanngurn S, et al, 2010 Immunohistological evidence for Wnt-signaling activation in Peutz-Jeghers polyposis. Pediatr Surg Int 26: 173-177.

43. Kikuchi A, Yamamoto H, 2008 Tumor formation due to abnormalities in the $\beta$-catenin-independent pathway of Wnt signaling. Cancer Sci 99: 202-208.

44. Liu C, Tu Y, Sun X, et al, 2010 Wnt/ beta-Catenin pathway in human glioma: expression pattern and clinical/ prognostic correlations. Clin Exp Med Aug 31 (e-pub ahead of print).

45. Inoki K, Corradetti MN, Guan KL, 2005 Dysregulation of TSC-mTOR pathway in human disease. Nat Gen 37: 19-24.

46. Van Slegtenhorst M, Nellist M, Nagelkerken B, et al, 1998 Interaction between hamartin and tuberin, the TSC1 and TSC2 gene products. Hum Mol Genet 7: 1053-1057.

47. Mita MM, Mita A, Rowinsky EK, 2003 Mammalian target of rapamycin: a new molecular target for breast cancer. Clin Breast Cancer 4: 126-137.

48. Bose S, Chandran S, Mirocha JM, Bose N, 2006 The akt pathway in human breast cancer: a tissue-array-based analysis. Mod Pathol 19: 238-245.

49. Shaw RJ, Bardeesy N, Manning BD, et al, 2004 The LKB-1 tumor suppressor negatively regulates mTOR signaling. Cancer Cell 6: 91-99.

50. Zakikhani M, Blouin MJ, Piura E, Pollak MN, 2010 Metformin and rapamycin have distinct effects on the AKT pathway and proliferation in breast cancer cells. Breast Cancer Res Treat published online: 05 February 2010.

51. Kuhajda FP, Pizer ES, Li JN, et al, 2000 Synthesis and antitumor activity of an inhibitor of fatty acid synthase. Proc Natl Acad Sci USA 97: 3450-3454.

52. Zhou G, Myers R, Li Y, et al, 2001 Role of AMP-activated protein kinase in mechanism of metfomin action. $\mathrm{J}$ Clin Invest 108: 1167-1174.

53. Xiang X, Saha AK, Wen R, et al, 2004 AMP-activated protein kinase activators can inhibit the growth of prostate cancer cells by multiple mechanisms. Biochem Biophys Res Commun 321: 161-167.

54. Jones RG, Plas DR, Kubek S, et al, 2005 AMP-activated protein kinase induces a p53-dependent metabolic checkpoint. Mol Cell 18: 283-293.

55. Rajawat YS, Bossis I, 2008 Autophagy in aging and in neurodegenerative disorders. Hormones (Athens) 7 : 46-61.

56. Giese A, Bjerkvig R, Berens M, Westphal M, 2003 Cost of migration: invasion of malignant gliomas and implications for treatment. J Clin Oncol 21: 1624-1636.
57. Rattan R, Giri S, Singh A, Singh I, 2006 5-Aminoimidazole-4-carboxamide-1-beta-D-ribofuranoside inhibits cancer cell proliferation in vitro and in vivo via AMP-activated protein kinase. J Biol Chem 280: 39582-39593.

58. Laderoute KR, Amin K, Calaogan JM, et al, 20065 `AMP-activated protein kinase (AMPK) is induced by low-oxygen and glucose deprivation conditions found in solid-tumor microenvironments. Mol Cell Biol 26: 5336-5347.

59. Shaw MM, Gurr WK, McCrimmon RJ, et al, 2007 5 'AMP-activated protein kinase alpha deficiency enhances stress-induced apoptosis in BHK and PC12 cells. J Cell Mol Med 11: 286-298.

60. Baumann P, Mandl-Weber S, Emmerich B, et al, 2007 Inhibition of adenosine monophosphate-activated protein kinase induces apoptosis in multiple myeloma cells. Anticancer Drugs 18: 405-410.

61. Vucicevic L, Misirkic M, Janetovic K, et al, 2009 AMPactivated protein kinase-dependent and -independent mechanisms underlying in vitro antiglioma action of compound C. Biochem Pharmacol 77: 1684-1693.

62. Wolf I, Sedetzki S, Catane R, et al, 2005 Diabetes mellitus and breast cancer. Lancet Oncol 6: 103-111.

63. Wolf I, Sedetzki S, Gluck I, et al, 2006 Association between diabetes mellitus and adverse characteristics of breast cancer at presentation. Eur J Cancer 42: 10771082.

64. Sachdev D, Singh R, Fujita-Yamaguchi Y, et al, 2006 Down-regulation of insulin receptor by antibodies against the type I insulin-like growth factor receptor: implication for anti-insulin-like growth factor therapy in breast cancer. Cancer Res 66: 2391-2402.

65. Goodwin PJ, Ennis M, Pritchard KI, et al, 2002 Fasting insulin and outcome in early-stage breast cancer: results of a prospective cohort study. J Clin Oncol 20: 42-51.

66. Millikan RC, Newman B, Tse CK, et al, 2008 Epidemiology of basal-like breast cancer. Breast Cancer Res Treat 109: 123-139.

67. Yang XR, Sherman ME, Rimm DL, et al, 2007 Differences in risk factors for breast cancer molecular subtypes in a population-based study. Cancer Epidemiol Biomarkers Prev 16: 439-443.

68. Sorlie T, Wang Y, Xiao C, et al, 2006 Distinct molecular mechanisms underlying clinically relevant subtypes of breast cancer: gene expression analyses across three different platforms. BMC genomics 7: 127.

69. Liu B, Fan Z, Edgerton SM, et al, 2009 Metformin induces unique biological and molecular responses in triple negative breast cancer cells. Cell Cycle 8: 20312040.

70. Alimova IN, Liu B, Fan Z, et al, 2009 Metformin inhibits breast cancer cell growth, colony formation and induces cell cycle arrest in vitro. Cell Cycle 6: 909-915.

71. Zhuang Y, Miskimins WK, 2008 Cell cycle arrest in metformin treated breast cancer cells involves activation 
of AMPK, downregulation of cyclin D1, and requires p27Kip1 or p21Cip1. J Mol Signal 3: 18-28.

72. Neve RM, Chin K, Fridlyand J, et al, 2006 A collection of breast cancer cell lines for the study of functionally distinct cancer subtypes. Cancer Cell 10: 515-527.

73. Phoenix KN, Vumbaca F, Claffey KP, 2009 Therapeutic metformin/AMPK activation promotes the angiogenic phenotype in the ER $\alpha$ negative MDA-MB-435 breast cancer model. Breast Cancer Res 113: 101-111.

74. Jiralerspong S, Giordano SH, Palla SL, et al, 2009 Metformin and pathologic complete responses to neoadjuvant chemotherapy in diabetic patients with breast cancer. J Clin Oncol 27: 3297-3302.

75. Garcia A, Tisman G, 2010 Metformin, B12, and enhanced breast cancer response to chemotherapy. J Clin Oncol 28: p.e19.

76. Hwang JT, Ha J, Park IJ, et al, 2007 Apoptotic effect of EGCG in HT-29 colon cancer cells via AMPK signal pathway. Cancer Lett 247: 115-121.

77. Hwang JT, Kim YM, Surh YJ, et al, 2006 Selenium regulates cyclooxygenase-2 and extracellular signalregulated kinase signaling pathways by activating AMPactivated protein kinase in colon cancer cells. Cancer Res 66: 10057-10063.
78. Degenhardt K, Mathew R, Beaudoin B, et al, 2006 Autophagy promotes tumor cells survival and restricts necrosis, inflammation and tumorigenesis. Cancer Cell 10: 51-64.

79. Koepsell H, Lips K, Volk C, 2007 Polyspecific organic transporters: structure, function, physiologic roles, and biopharmaceutical implications. Pharm Res 24: 1227 1251.

80. Shu Y, Sheardown S, Brown C, et al, 2007 Effect of genetic variation in the organic cation transporter 1 (OCT1) on metformin action. J Clin Invest 117: 14221431.

81. Nies At, Koepsell H, Winter S, et al, 2009 Expression of organic cation transporterts OCT1(SLA22A1) and OCT3 (SLC22A3) is affected by genetic factors and cholestasis in human liver. Hepatology 50: 1227-1240.

82. Tzvetkov MV, Vormfelde SV, Balen D, et al, 2009 The effects of genetic polymorphisms in the organic cation transporters OCT1, OCT2, and OCT3on the renal clearance of metformin. Clin Pharmacol Therap 86: 299-306.

83. Algire C, Zakikhani M, Blouin MJ, et al, 2008 Metformin attenuates the stimulatory effect of a high-energy diet on in vivo LLCI carcinoma growth. Endocr Relat Cancer 15: 833-839. 